
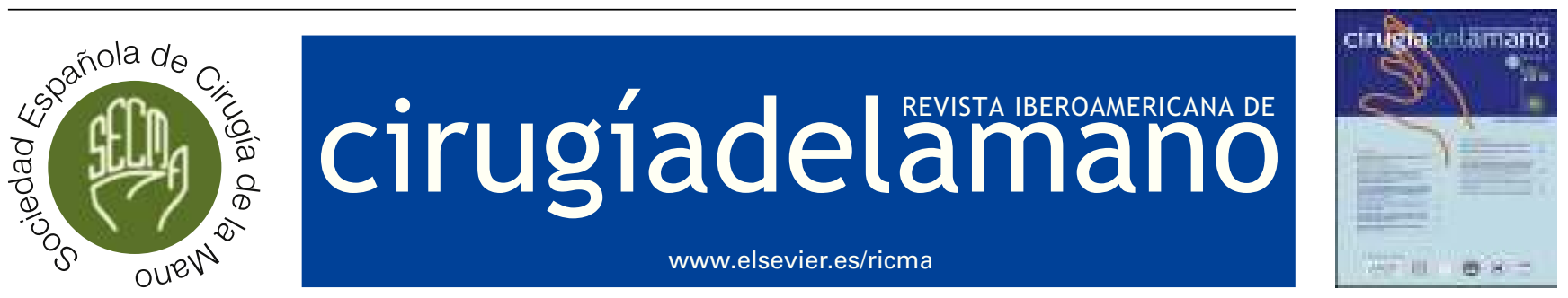

TÉCNICA QUIRÚRGICA

\title{
Técnica de reinserción foveal artroscópica sin nudos del fibrocartílago triangular con visión directa de la articulación radiocubital distal
}

\author{
V. Carratalá Baixaulia,*, F.J. Lucas García ${ }^{a}$, C.A. González Jofré ${ }^{b}$, \\ R. Carratalá Baixauli ${ }^{\mathrm{c}}$ y E. Sánchez Alepuz ${ }^{\mathrm{a}}$ \\ a Servicio de Cirugía Ortopédica y Traumatología, Unión de Mutuas, Valencia, España \\ b Servicio de Traumatología, Hospital Carlos Van Buren, Mutual de Seguridad, V Región, Valparaíso, Chile \\ c Servicio de Cirugía Ortopédica y Traumatología, Hospital Clínico Universitario, Valencia, España
}

Recibido el 25 de enero de 2016; aceptado el 24 de febrero de 2016

Disponible en Internet el 2 de abril de 2016

\section{PALABRAS CLAVE}

Fibrocartílago

triangular;

Reinserción foveal;

Artroscopia

de muñeca

\section{KEYWORDS}

Triangular

fibrocartilage;

Foveal reattachment;

Wrist arthroscopy

\begin{abstract}
Resumen El fibrocartílago triangular es una estructura de alta relevancia en la estabilidad de la articulación radiocubital distal. Su componente profundo es el más importante en esta función. Por ello, cuando clínicamente se precisa recuperar esta estabilidad, se debe apuntar a una técnica quirúrgica que permita reestablecer lo más anatómicamente este componente. Presentamos una técnica artroscópica, sin nudos, que mediante 2 portales accesorios a los radiocarpianos, el portal distal dorsal de la articulación radiocubital distal y el portal distal volar radioulnar, permite reinsertar el fibrocartílago triangular en su punto de origen, de forma anatómica mediante visión directa durante todo el procedimiento. Posibilita además cruentar la huella de inserción aportando factores biológicos autólogos, mejorando la interfase huesofibrocartílago y analizar el estado local del cartílago articular, lo que condicionará la posterior indicación quirúrgica.

(c) 2016 SECMA. Publicado por Elsevier España, S.L.U. Este es un artículo Open Access bajo la licencia CC BY-NC-ND (http://creativecommons.org/licenses/by-nc-nd/4.0/).
\end{abstract}

Foveal arthroscopic reattachment technique with knotless triangular fibrocartilage with direct view of the distal radio-ulnar joint

Abstract The triangular fibrocartilage is a structure of high importance to the stability of the distal radio-ulnar joint. The deep component of the triangular fibrocartilage is the most important in this role. Therefore, when it is required to recover the stability, a surgical technique needs to be performed for anatomical restoration. A knotless arthroscopic technique is presented, using two access portals in addition to the radiocarpal portals. This technique uses the distal dorsal portal and distal volar portal of the distal radio-ulnar joint. Through these portals

\footnotetext{
* Autor para correspondencia.

Correo electrónico: vteca@ono.com (V. Carratalá Baixauli).
} 
reattachment of the triangular fibrocartilage into the ulnar fovea can be performed anatomically by direct vision during the whole procedure. This technique also allows the debridement of the footprint, improving the bone-fibrocartilage interface and the visualisation of the status of the articular cartilage, which will determine the posterior surgical indication

(C) 2016 SECMA. Published by Elsevier España, S.L.U. This is an open access article under the CC BY-NC-ND license (http://creativecommons.org/licenses/by-nc-nd/4.0/).

\section{Introducción}

El fibrocartílago triangular (FCT) es una estructura compuesta por tejido fibrocartilaginoso y ligamentos entre el radio, cúbito, semilunar y piramidal ${ }^{1}$. Cumple un importante papel permitiendo el movimiento de rotación del radio sobre el cúbito, distribuye las cargas a través de la columna ulnocarpiana y contribuye en la estabilidad de la articulación ulnocarpiana y la radiocubital distal (ARCD), siendo el componente profundo del fibrocartílago triangular (CP-FCT) el más relevante en esta función ${ }^{2-4}$.

Lesiones del FCT pueden, en diversos grados, afectar a la estabilidad de la ARCD generando además de dolor, disminución de la funcionalidad de la muñeca e incluso conducir a una degeneración progresiva de la articulación.

Existen varias técnicas abiertas, o combinando la artroscopia con mini-incisiones, para la reparación del CP-FCT mostrando resultados satisfactorios, pero que no visualizan directamente la fóvea durante el procedimiento, realizando una reinserción que puede distar del lugar más idóneo y en ocasiones necesitar de la ayuda de la radioescopia intraoperatoria ${ }^{2,5,6}$.
La presente publicación tiene como objetivo describir la técnica artroscópica que empleamos y que permite una reinserción foveal anatómica y sin nudos del CP-FCT realizada completamente por artroscopia empleando, además de los portales convencionales ${ }^{7}$, los portales de acceso a la ARCD para situar y objetivar bajo visión directa el lugar de reinserción exacto del CP-FCT en la fóvea.

\section{Anatomía quirúrgica}

La parte distal ulnar del FCT consta de 3 componentes: el ligamento triangular proximal, la hamaca distal y el ligamento colateral ulnar ${ }^{7-10}$. La hamaca distal y el ligamento colateral forman el componente superficial, que se inserta directamente en la estiloides ulnar. Por su parte, el ligamento proximal, con los ligamentos radioulnares volar y dorsal, forman parte del CP-FCT, que se inserta directamente en la fóvea, adyacente a la superficie articular distal del cúbito (fóvea ulnar) ${ }^{2,7,8}$. Estudios anatómicos y biomecánicos $^{2-4}$ han señalado al CP-FCT como el componente de mayor relevancia en la estabilidad, comparado con el componente superficial. De esto deriva la necesidad de

Tabla 1 Clasificación de las lesiones periféricas del FCT de Atzei y Luchetti

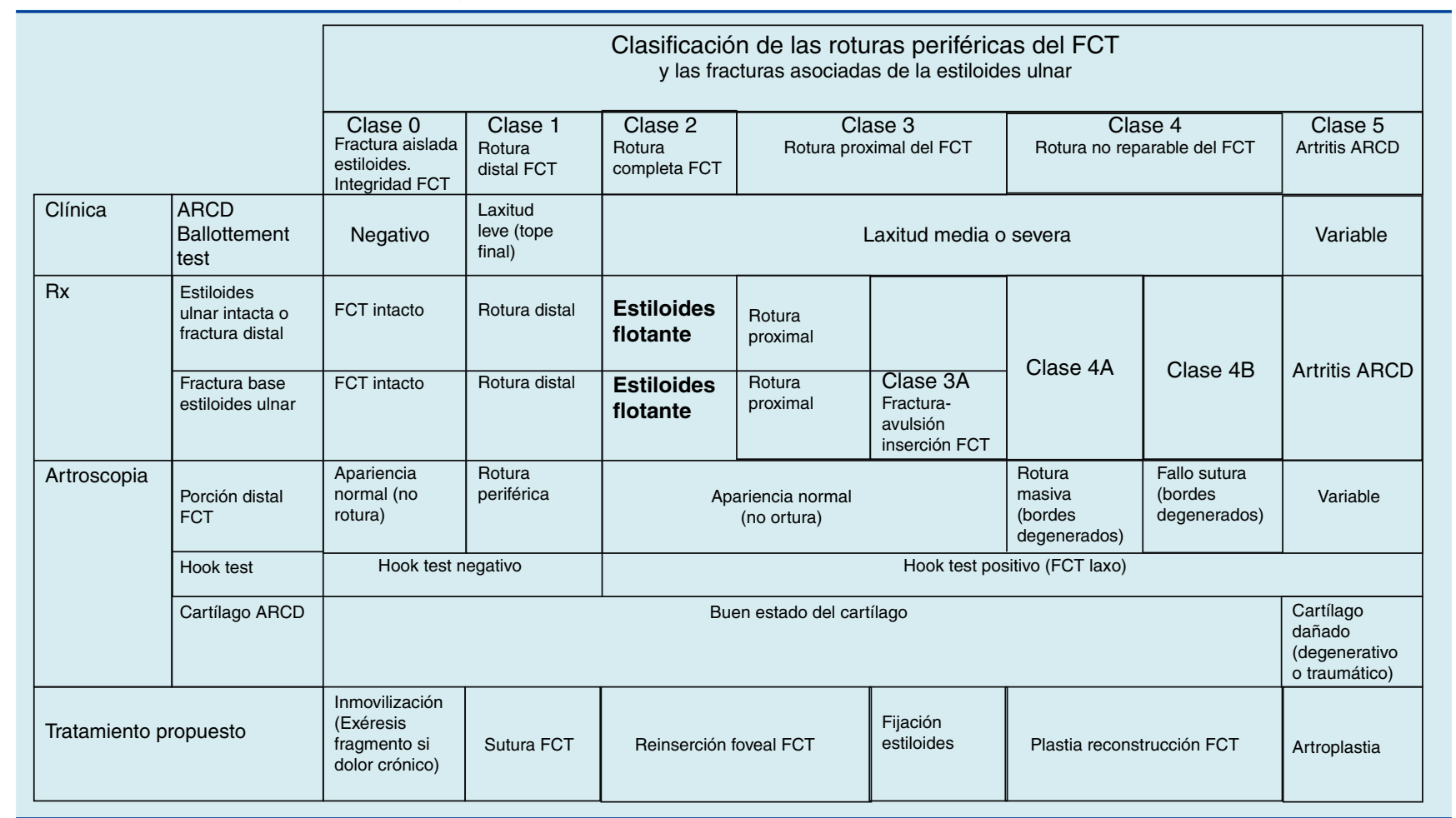

En negrita, subestadios de la patología. 
una localización lo más precisa posible de la fóvea durante la reinserción del FCT, buscando reestablecer la anatomía de la ARCD.

\section{Indicaciones}

La indicación de la técnica sería para aquellos casos con una desinserción foveal aguda o subaguda del CP-FCT con suficiente calidad de tejido del FCT, en los que aparece una inestabilidad de la ARCD, clase 2 y 3 de la clasificación de Atzei y Luchetti ${ }^{11}$ (tabla 1), con manifestaciones clínicas y que se confirma mediante la exploración artroscópica.

Las ventajas de este procedimiento artroscópico son:

1. Permite la objetivación de la desinserción foveal bajo la visión directa mediante la exploración de la articulación radiocubital distal, así como también valorar el estado del cartílago de esta, esencial para una correcta indicación quirúrgica y un mejor resultado clínico.

2. Posibilita la limpieza de la fóvea de los restos fibróticos de material existente, cruentar el lecho de inserción estimulando biológicamente una mejor interfase huesoFCT, aportando también factores biológicos provenientes directamente de la zona subyacente.

3. Identifica la localización exacta de la fóvea permitiendo observar directamente el punto en que se ancla la reparación del FCT para así respetar lo máximo posible la isometría y biomecánica de este complejo9 .

4. Realiza una reinserción sin nudos, evitando la presencia de un cuerpo extraño intraarticular que en ocasiones puede provocar irritación a nivel de articulación ulnocarpiana.

\section{Contraindicaciones}

Las lesiones no reparables (clase 4 de la clasificación de Atzei) y las lesiones con cambios artrósicos en la articulación

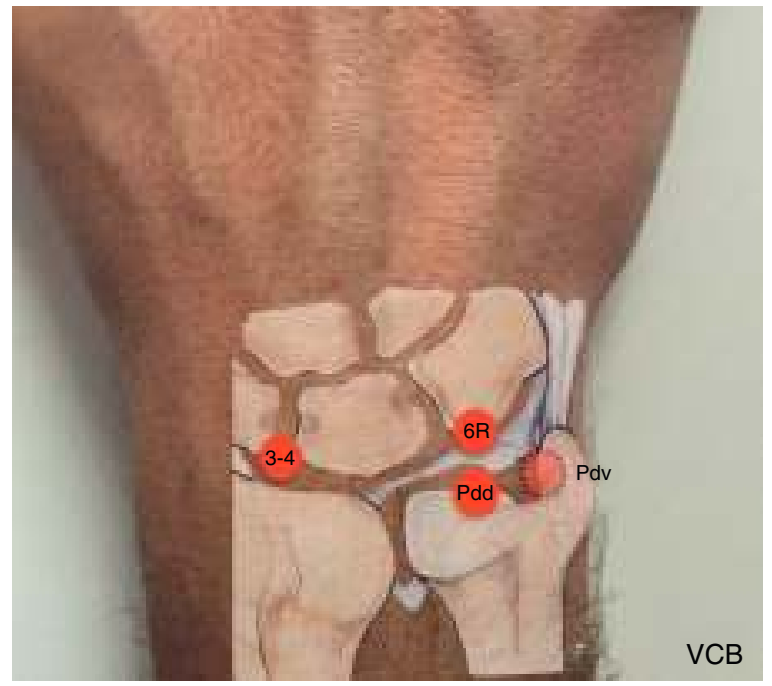

Figura 1 Portales empleados en la técnica. Portales radiocarpianos: 3-4 y 6R. Portales de acceso a la ARCD: pdd-RCD y pdv-RU.

radiocubital distal (clase 5 de Atzei) ${ }^{11}$, además de cualquier circunstancia que contraindicara la artroscopia de muñeca.

\section{Técnica quirúrgica}

\section{Paso 1: posicionamiento y portales}

Situamos al paciente en decúbito supino, en mesa de mano, con el miembro superior bajo tracción de muñeca con el sistema Acumed Arc Wrist Tower (Acumed, Hillsboro, OR) y utilizamos una óptica de artroscopia 2,4 mm (Arthrex, Naples, FL). Los portales que empleamos para esta técnica serán los radiocarpianos 3-4 y 6R ${ }^{12}$, el portal dorsal distal de la radiocubital distal ${ }^{13}$ (pdd-RCD) y el portal distal volar radioulnar $^{14}$ (pdv-RU) (fig. 1).

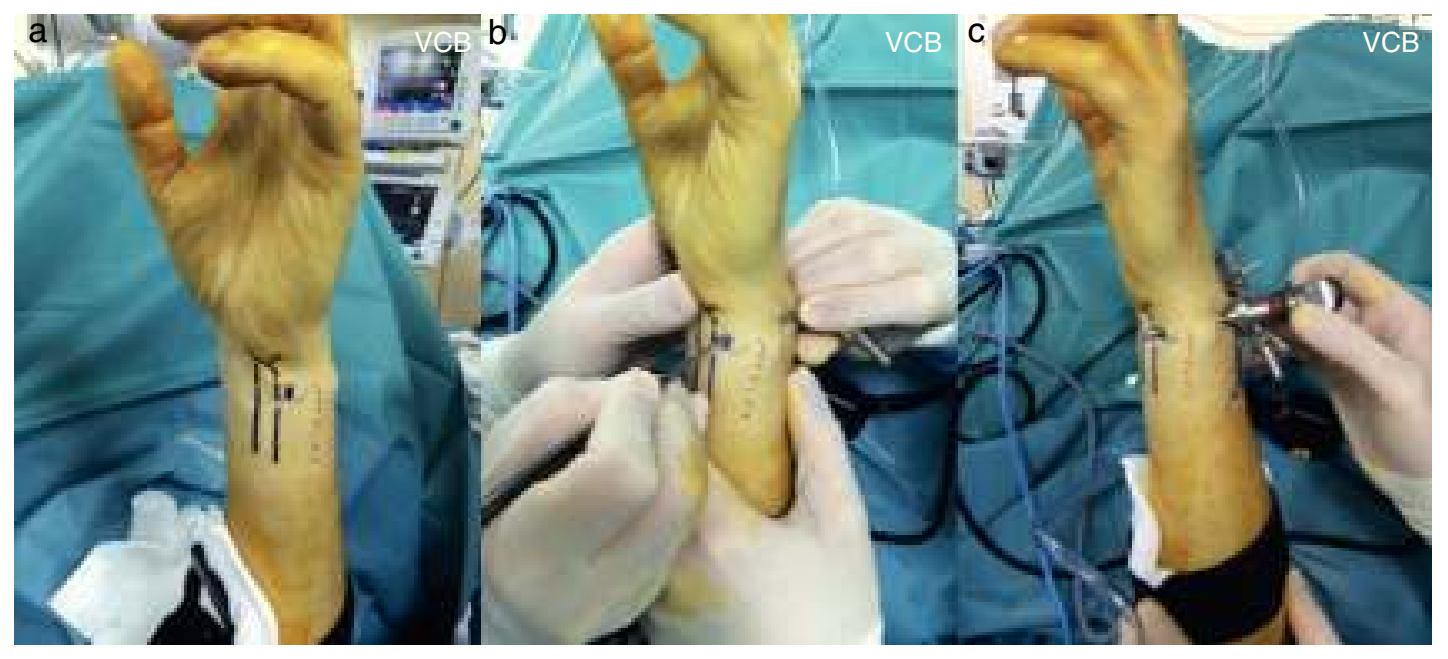

Figura 2 Realización del pdv-RU mediante técnica de dentro a fuera. a) Imagen con el pdv-RU marcado adyacente al borde ulnar del tendón flexor carpi ulnaris. b) Incisión en el punto del portal tras comprobar, empujando el trócar, que la posición es la correcta. c) Extracción de la cánula a través de la incisión realizada completando la realización del portal. 


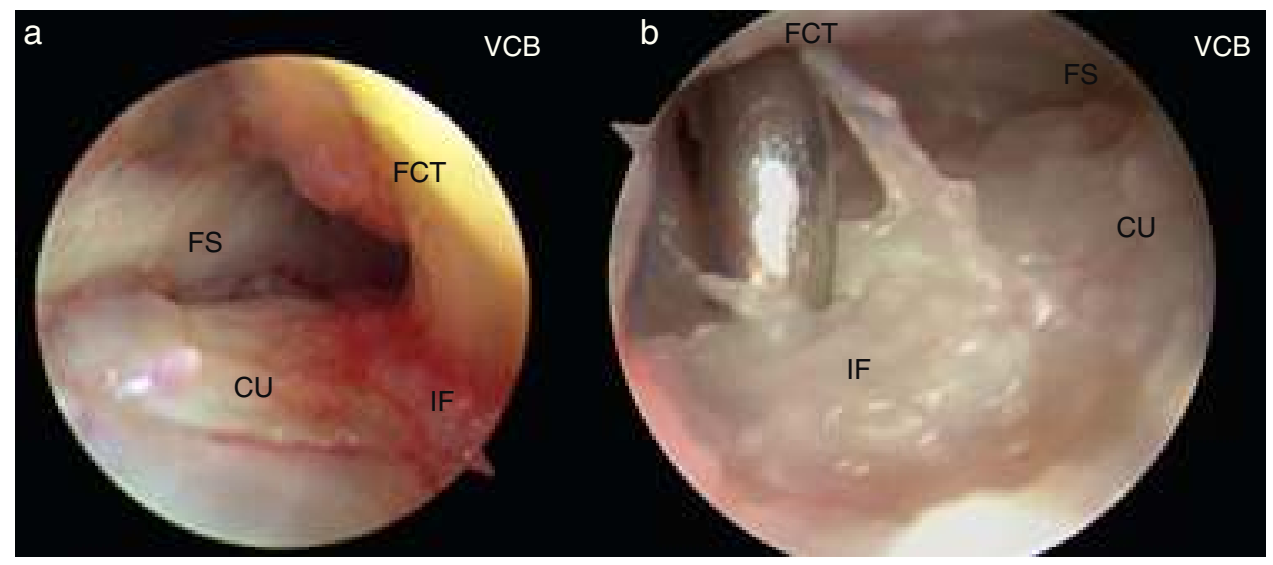

Figura 3 Diagnóstico directo de la lesión foveal del FCT. a) Imagen de inserción foveal sana, visión desde el pdd-RCD. b) Imagen de una desinserción foveal del FCT, visión desde el pdd-RCD.

CU: cabeza ulnar; FCT: fibrocartílago triangular; FS: fosa sigmoidea; IF: inserción foveal.
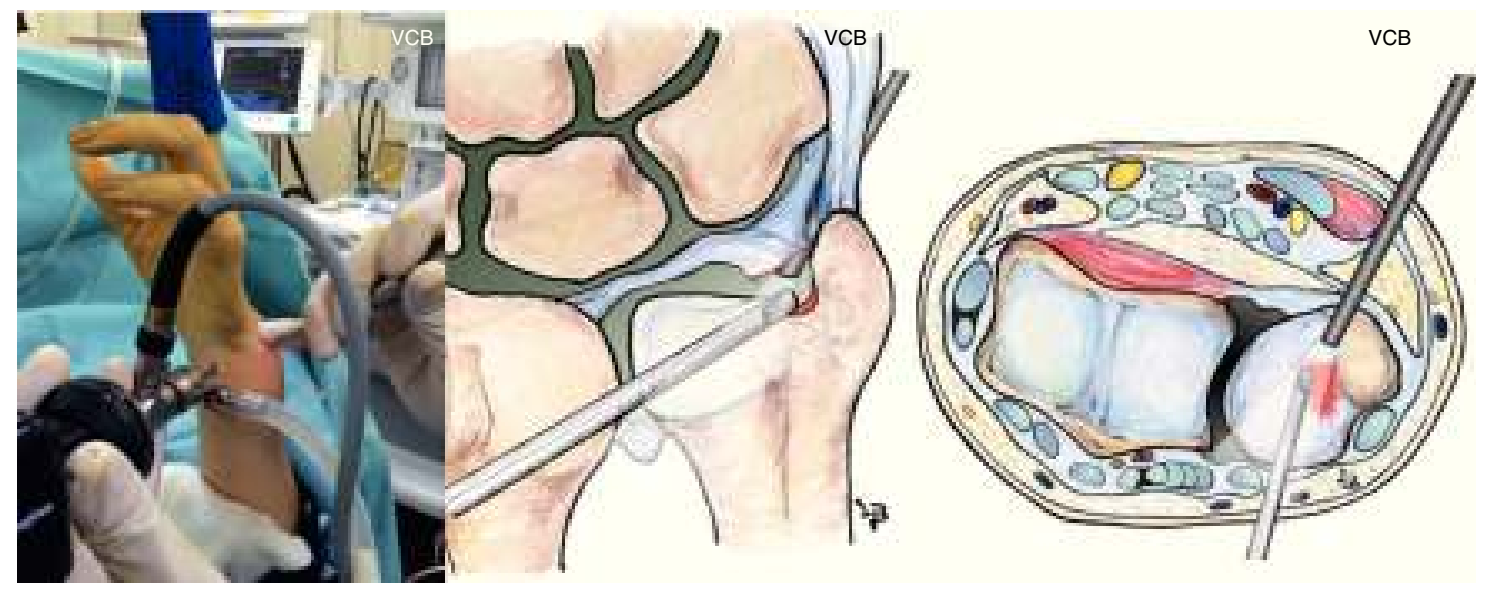

Figura 4 Limpieza y cruentación de la huella de inserción del CP-FCT en la fóvea. Óptica en el pdv-RU y sinoviótomo en el pdd-RCD. Es importante una buena limpieza del espacio para poder tener una visualización óptima del procedimiento.

Establecimiento de los portales ARCD:

Pdd-RCD: se ubica de 6 a $8 \mathrm{~mm}$ proximales al portal $6 \mathrm{R}$ en la parte superior de la cabeza ulnar, pero por debajo del FCT. Para establecer el portal, con visión directa desde el portal 3-4 introducimos una aguja proximalmente al portal
6R comprobando que nos encontramos por debajo del disco del FCT ${ }^{13,15}$.

Pdv-RU: se localiza a unos 5-10 mm proximales al pliegue proximal de la muñeca, justo en el borde ulnar del tendón flexor carpi ulnaris y radial a la rama cutánea dorsal del

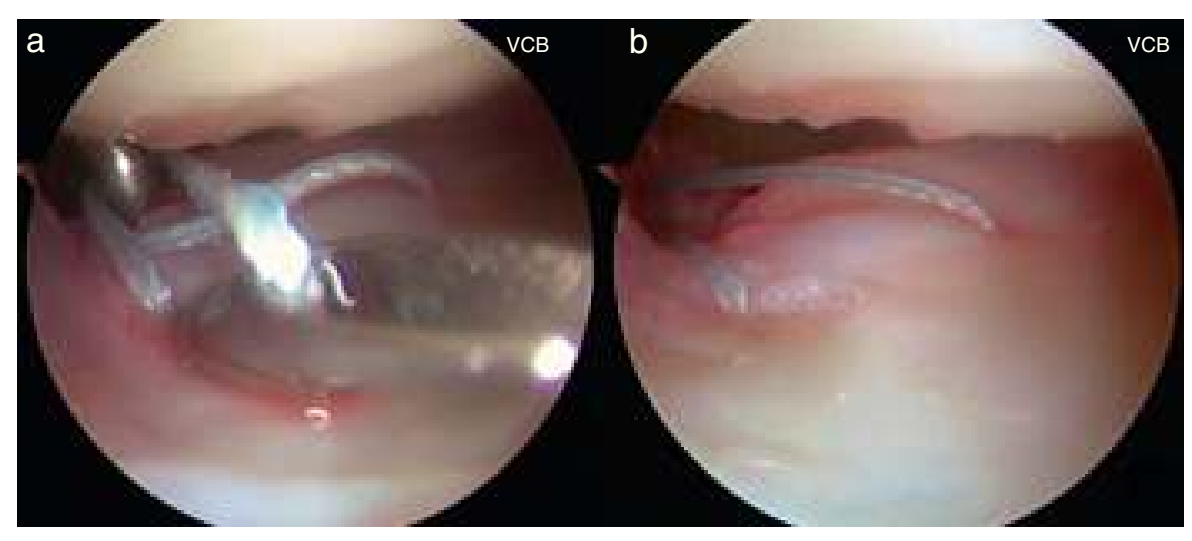

Figura 5 Paso de la sutura de Fiber Stick 2/0 (Arthrex, Naples, FL) a través del FCT. Visión desde el portal 3-4. a) Paso del segundo cabo de la sutura por la porción dorsal del FCT y recuperación de la misma a través del portal 6R. b) Ambos cabos de la sutura se han pasado a través del FCT, uno en borde volar y otro en el dorsal. Los 2 cabos son recuperados por el portal 6R. 


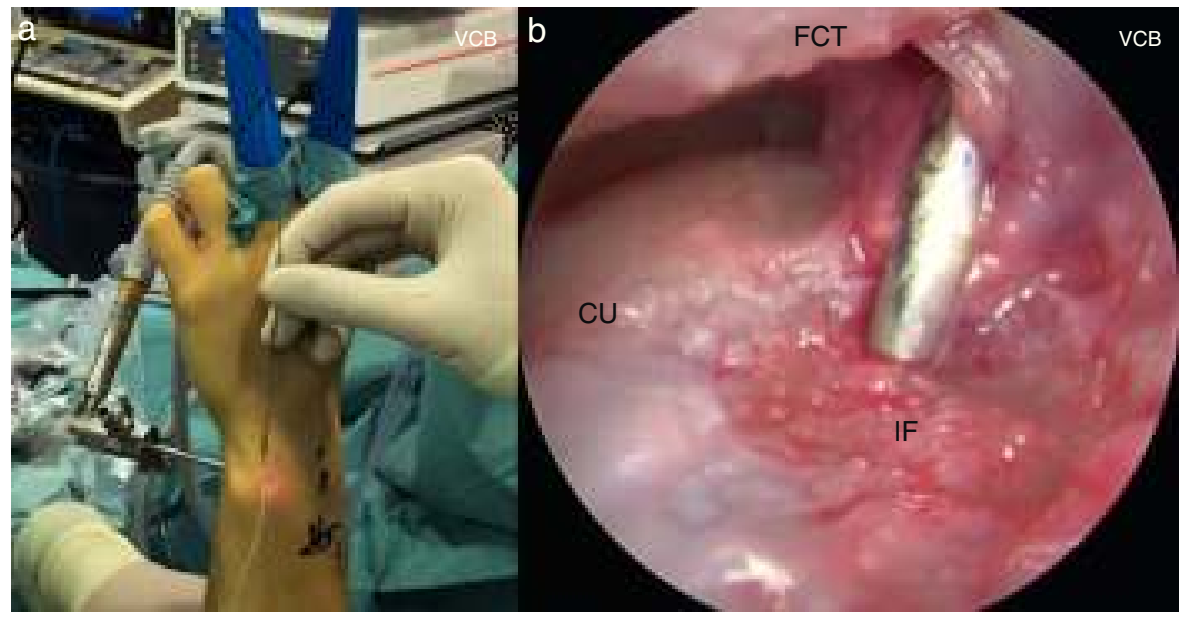

Figura 6 a) Visión externa, la óptica en el pdv-RU. Desde el portal 6R se coloca la aguja guía y se localiza con visión directa su posición en la fóvea. b) Visión artroscópica del mismo paso desde el pdv-RU. CU: cabeza ulnar; FCT: fibrocartílago triangular; IF: inserción foveal.

nervio ulnar. La estiloides ulnar marca el punto distal del portal. Para facilitar la localización exacta, realizamos el portal con una técnica de transiluminación a través del pdd-RCD ${ }^{13}$. Este portal puede establecerse mediante técnica de fuera a dentro con una aguja o mediante técnica de dentro a fuera mediante la extracción del trócar en el punto del portal previamente marcado (fig. 2).

\section{Paso 2: exploración y diagnóstico}

Iniciamos el procedimiento con la artroscopia radiocarpiana, explorando el borde ulnar y descartando otras entidades concomitantes. Un Hook test sign positivo ${ }^{8}$ nos confirma la sospecha diagnóstica de desinserción foveal del FCT.

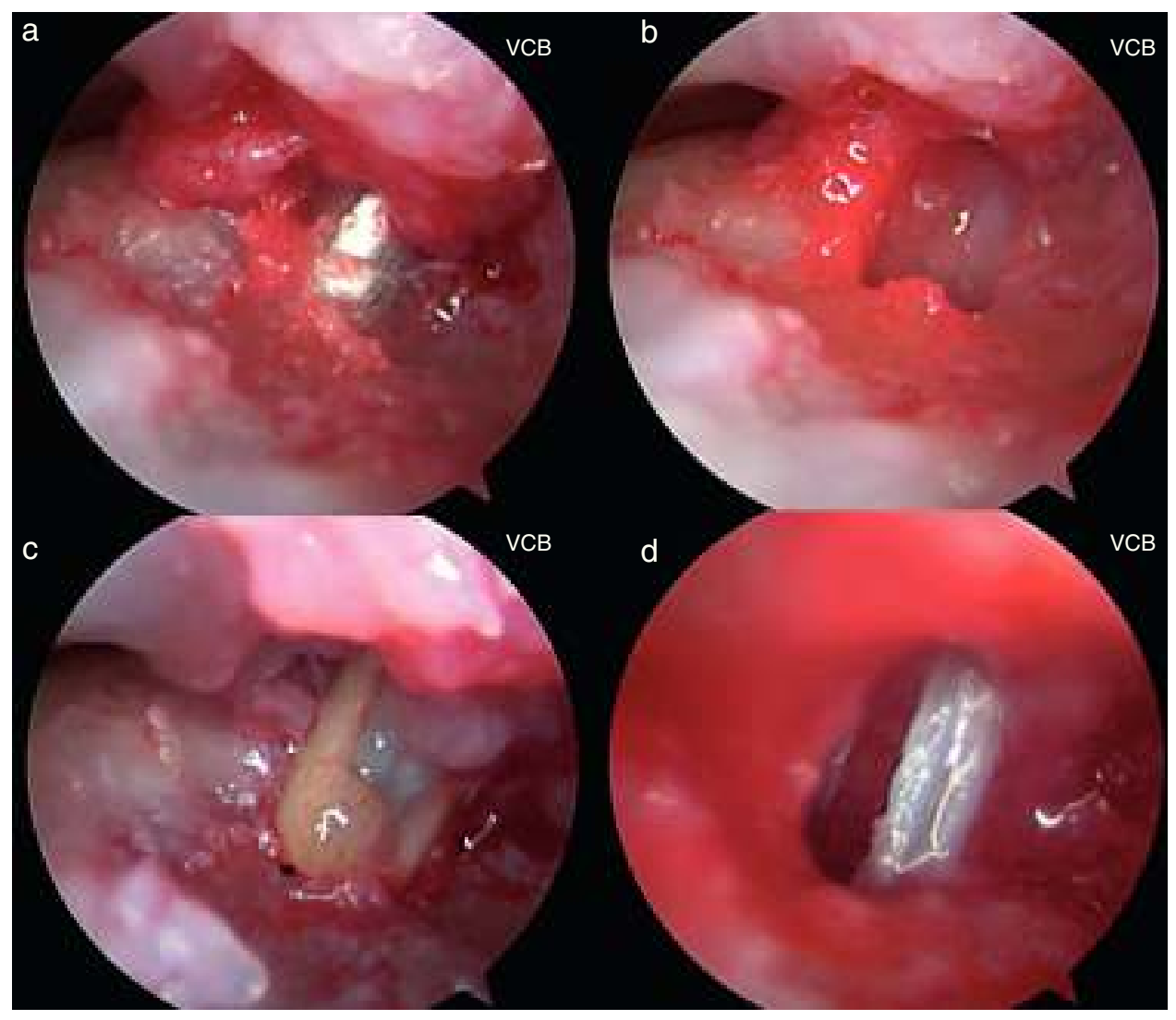

Figura 7 Visión artroscópica desde el pdv-RU. Secuencia del brocado y colocación del implante. a) Paso de la broca a través de la aguja guía desde el portal 6R. b) Perforación realizada en el lecho foveal. c) Introducción del implante desde el portal 6R. d) Cierre del espacio de visión que se produce al realizar la impactación del implante y finalizar la reinserción foveal del FCT. 
Realizamos entonces los portales que nos darán acceso a la ARCD tal y como se han descrito previamente. Iniciamos la artroscopia de la ARCD desde el pdd-RCD, confirmamos completamente la lesión y localizamos el punto exacto de la desinserción del FCT a nivel de la fóvea ulnar (fig. 3 y vídeo). El pdv-ARCD en un primer tiempo lo empleamos como portal de trabajo, introduciendo el sinoviótomo para limpiar el espacio entre el borde proximal del FCT y la cabeza ulnar, colocando luego, por este mismo portal, la óptica para completar la exploración.

\section{Paso 3: preparación de la huella de inserción foveal}

Con la óptica en el pdv-RU y el sinoviótomo en el pdd-RCD se realiza la localización, limpieza y cruentación del lecho donde realizaremos la reinserción. Este paso es importante para estimular el potencial de cicatrización de la reparación (fig. 4 y vídeo). Una vez limpio el espacio, la localización de la fóvea es más sencilla, mediante visión directa artroscópica, pudiendo ver los restos de la inserción del CP-FTC y en ocasiones avulsiones osteocondrales en el punto de la desinserción.

\section{Paso 4: artroscopia radiocarpiana y paso de suturas a través del fibrocartílago triangular}

Con la óptica en el portal 3-4 y visión sobre el FCT, pasamos una sutura Fiber Stick 2/0 (Arthrex, Naples, FL) por 2 puntos a través del FCT. Este paso puede realizarse con la ayuda de una aguja espinal introduciendo la sutura en su interior o empleando un Micro suture Lasso curvo $70^{\circ}$ (Arthrex, Naples, $\mathrm{FL}$ ) introducido desde el portal 6R.

El primer cabo de la sutura debe atravesar la porción volar del FCT (ligamento radioulnar volar), siendo recuperado por el portal 6R con la ayuda de un grasper o un mosquito. Retirando la aguja espinal y sin salir de la cápsula articular, volvemos a atravesar el FCT, en esta ocasión en la porción más dorsal (ligamento radioulnar dorsal), recuperando el segundo cabo también por el portal 6R con la ayuda de un gancho palpador (fig. 5 y vídeo). De esta

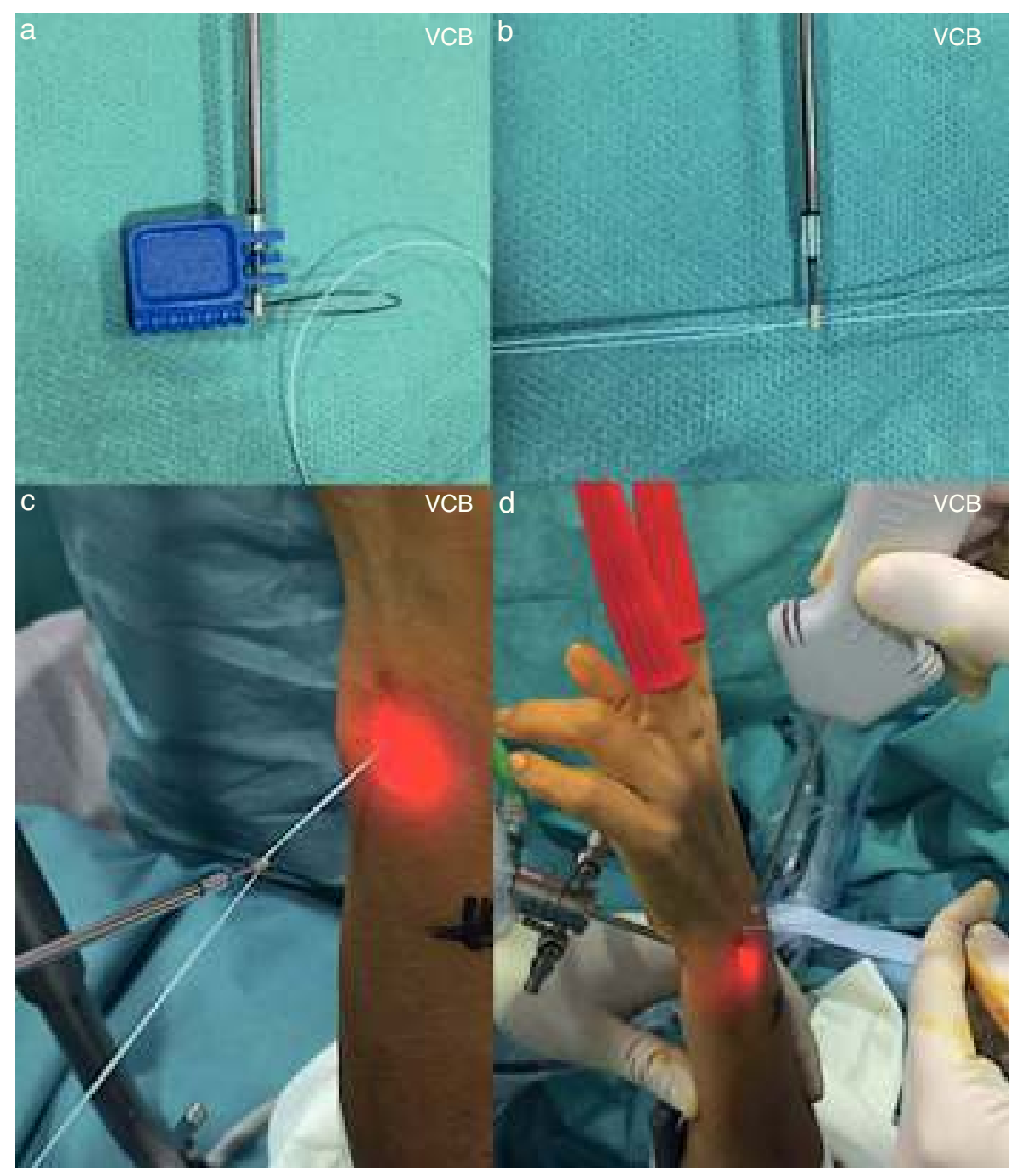

Figura 8 a y b) Paso de los cabos de la sutura a través del orificio del implante. c y d) Introducción del implante por el portal 6R mientras mantenemos la visión desde el pdv-RU. 


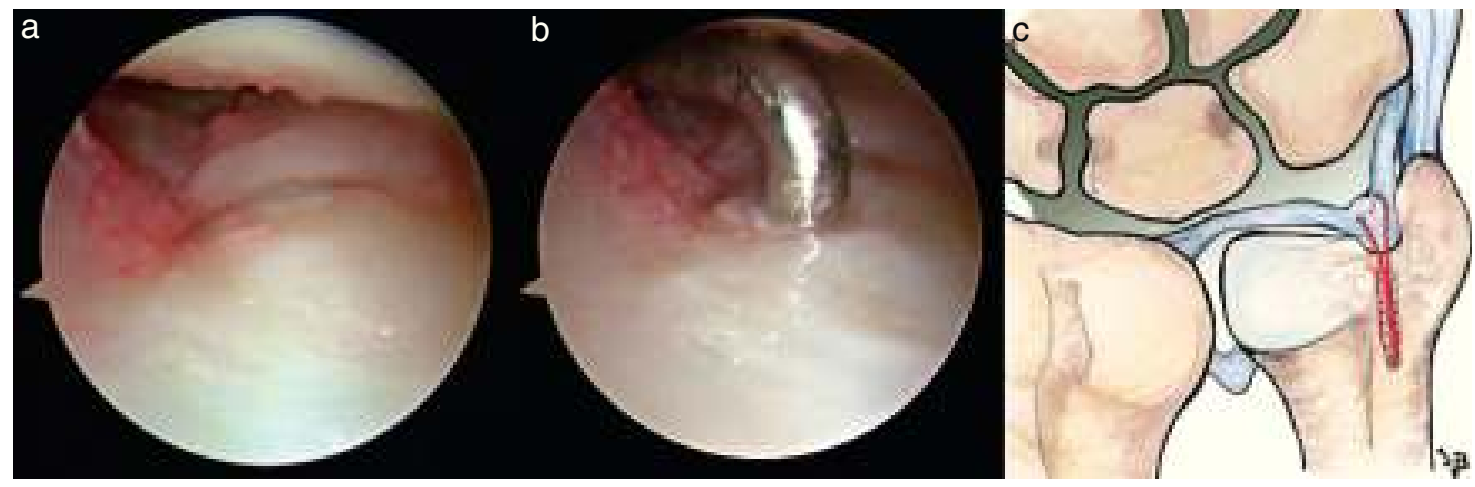

Figura 9 a y b) Visión desde el portal 3-4. Comprobación del resultado de la reparación y de la tensión del FCT. c) Resultado final de la reinserción foveal sin nudos del FCT.

forma tenemos una sutura que pasa por 2 puntos del tejido del FCT y los 2 cabos recuperados en el portal $6 \mathrm{R}$.

\section{Paso 5: colocación de guía y brocado}

Con la óptica en portal dv-RU, se localiza la fóvea y se introduce la aguja guía desde el portal 6R (fig. 6). Esta debe pasar a través del defecto del FCT en la inserción capsular ulnar y se coloca en la huella de inserción foveal. Posteriormente se introduce la broca canulada de $1,8 \mathrm{~mm}$ del sistema Mini BioPushLock $^{\circledR}$ (Arthrex, Naples, FL) desde el portal 6R y se realiza el brocado en el punto de inserción. En todo este proceso mantenemos una visión artroscópica directa desde el portal dv-RU (fig. 7a,b y vídeo).

\section{Paso 6: colocación del implante}

Se pasan las 2 suturas enhebrándolas al implante (fig. 8). Se retira la aguja guía y se introduce el implante de $2,5 \mathrm{~mm}$ Mini BioPushLock ${ }^{\circledR}$ (Arthrex, Naples. FL) a través del portal $6 \mathrm{R}$, descendiendo el dispositivo al lugar de la perforación en la fóvea y colocando el implante bajo visión directa (fig. 7c, d y vídeo). Debe mantenerse la tensión de las suturas en el momento de la colocación del implante manteniendo la muñeca en posición neutra. Al impactar el implante, la visión en el espacio de la ARCD se va reduciendo a la vez que reinsertamos el FCT a la fóvea ulnar. Se comprueba el resultado final y la tensión del FCT desde el espacio radiocarpiano (fig. 9 y vídeo).

\section{Complicaciones}

Las principales complicaciones que pueden aparecer intraoperatoriamente con esta técnica son lesiones condrales iatrogénicas con el establecimiento de los portales de la $A R C D$, lesiones del paquete neurovascular ulnar o de los múltiples nervios cutáneos ulnares que recorren la zona ${ }^{16}$, la rotura o desgarro del FCT al pasar la sutura a través de él y la fractura de la cabeza ulnar o la pérdida de fijación del implante debido a un brocado brusco y/o excesivo. En el postoperatorio, la complicación más importante es la recurrencia de la lesión inicial, debido a la distensión o rotura de la reparación por una movilización demasiado precoz.

\section{Postoperatorio}

Tras la cirugía, se coloca una férula braquial en supinación durante 4 semanas que se sustituye por una ortesis antebraquial durante 2 semanas más, permitiendo la movilidad de los dedos y codo. Tras las 6 semanas de inmovilización se remite para inicio del protocolo de rehabilitación.

\section{Conflicto de intereses}

Los autores declaran no tener ningún conflicto de intereses.

\section{Anexo. Material adicional}

Se puede consultar material adicional a este artículo en su versión electrónica disponible en doi:10.1016/j.ricma. 2016.02.002.

\section{Bibliografía}

1. Palmer AK, Werner FW. The triangular fibrocartilage complex of the wrist anatomy and function. J Hand Surg Am. $1981 ; 6: 153-62$.

2. Kleinman WB. Stability of the distal radioulna joint: Biomechanics, pathophysiology, physical diagnosis, and restoration of function what we have learned in 25 years. J Hand Surg Am. 2007;32:1086-106.

3. Af Ekenstam F, Hagert CG. Anatomical studies on the geometry and stability of the distal radio ulnar joint. Scand J Plast Reconstr Surg. 1985;19:17-25.

4. Haugstvedt JR, Berger RA, Nakamura T, Neale P, Berglund L, An KN. Relative contributions of the ulnar attachments of the triangular fibrocartilage complex to the dynamic stability of the distal radioulnar joint. J Hand Surg Am. 2006;31:445-51.

5. Sennwald GR, Lauterburg M, Zdravkovic V. A new technique of reattachment after traumatic avulsion of the TFCC at its ulnar insertion. J Hand Surg Br. 1995;20:178-84.

6. Iwasaki N, Minami A. Arthroscopically assisted reattachment of avulsed triangular fibrocartilage complex to the fovea of the ulnar head. J Hand Surg Am. 2009;34:1323-6. 
7. Atzei A. New trends in arthroscopic management of type 1-B TFCC injuries with DRUJ instability. J Hand Surg Eur. 2009;34:582-91.

8. Atzei A, Rizzo A, Luchetti R, Fairplay T. Arthroscopic foveal repair of triangular fibrocartilage complex peripheral lesion with distal radioulnar joint instability. Tech Hand Up Extrem Surg. 2008;12:226-35.

9. Nakamura T, Makita A. The proximal ligamentous component of the triangular fibrocartilage complex. J Hand Surg Br. 2000;25:479-86.

10. Nakamura T, Yabe Y, Horiuchi Y. Functional anatomy of the triangular fibrocartilage complex. J Hand Surg Br. 1996;21:581-6.

11. Atzei A, Luchetti R. Foveal TFCC tear classification and treatment. Hand Clin. 2011;27:263-72.
12. Lucas FJ, Carratalá V, Sánchez E. Generalidades, portales y anatomía artroscópica. Rev Esp Artrosc Cir Articul. 2014;21: $5-13$.

13. Slutsky DJ. Wrist arthroscopy portals. En: Slutsky DJ, Nagel DJ, editores. Techniques in hand and wrist arthroscopy. Elsevier: Philadelphia; 2007. p. 2-17.

14. Atzei ALR, Carita E, Papin Zorli I, Cugola L. Arthroscopically assisted foveal reinsertion of peripheral avulsions of the TFCC. J Hand Surg Br. 2005;30 Suppl. 1:40.

15. Slutsky DJ. Arthroscopic evaluation of the foveal attachment of the triangular fibrocartilage. Hand Clin. 2011;27:255-61.

16. Martin CH, Seiler JG 3rd, Lesesne JS. The cutaneous innervation of the palm: An anatomic study of the ulnar and median nerves. J Hand Surg Am. 1996;21:634-8. 